\title{
Conflitos no relacionamento entre cuidadores e idosos: 0 olhar do cuidador
}

\section{Conflidsontherelationship betweencaregiversandtheeddely: thecaregivers' viexpaint}

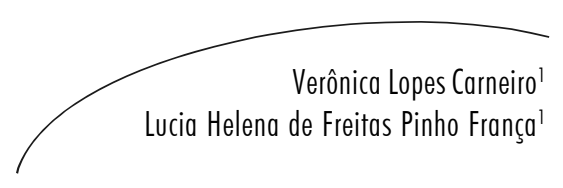

Este estudo investigou os conflitos no relacionamento entre os cuidadores e seus idosos, na visão de 100 cuidadores, residentes no Estado do Rio de Janeiro, e que preencheram um questionário sob a forma de entrevista. O estado civil do idoso e o nível de escolaridade do cuidador foram avaliados como possíveis variáveis que poderiam influenciar a frequência dos conflitos. Alguns itens do instrumento de solidariedade entre idosos e cuidadores de Lowestein, Katz, MehlhausenHassoen e Prilutzky (2005) foram adaptados e utilizados para construir e validar uma Escala de Frequência de Conflitos Percebidos entre o Cuidador e o Idoso (ECPCI), tipo Likert, variando de 1 (sempre) a 5 (nunca). A ECPCI apresentou excelente consistência teórica $(\alpha=0,91)$, e de sua análise fatorial emergiram duas dimensões: aborrecimentos no dia a dia $(\alpha=0,91)$ e criticismo e conflitos $(\alpha=0,83)$. $\mathrm{O}$ criticismo dos mais velhos com os cuidadores apresentou a maior frequência, e a mais baixa foi o aborrecimento em ter que cuidar dos idosos. Os idosos que não tinham parceiros apresentaram mais conflitos com os cuidadores do que os idosos que viviam com parceiros $(\mathrm{t}=2,76$; $\mathrm{p}<0,05)$ Nenhuma correlação foi encontrada entre o nível de escolaridade do cuidador e seus conflitos com o idoso. Sugerem-se estratégias para a redução destes conflitos, como estimular as organizações a oferecerem cursos para a melhoria das habilidades de comunicação interpessoal entre cuidadores e idosos, bem como a profissionalização desta emergente e importante carreira para a sociedade brasileira.

\footnotetext{
Universidade Salgado de Oliveira, Conselho de Ensino, Pesquisa e Extensão, Programa de Pósgraduação em Psicologia. Niterói, RJ, Brasil.

Artigo baseado na dissertação de Mestrado de Verônica Lopes Carneiro, defendida em 2009 na Universidade Salgado de Oliveira-Universo.
}

Palavras-chave: Relacionamento interpessoal. Cuidadores. Idosos. Escala de conflitos. 


\section{Abstract}

This study investigated the conflicts between caregivers and the elderly in their care, from the viewpoint of 100 caregivers, living in the State of Rio de Janeiro who filled in a questionnaire, during an interview. The marital status of the elderly and the level of schooling of the caregivers were assessed as possible variables influencing the frequency of the conflicts. Some items of an instrument of a study of solidarity between elderly and their caregivers by Lowestein, Katz, Mehlhausen-Hassoen e Prilutzky (2005) were used to build and validate the Scale of Perceived Conflicts between Caregivers and the Elderly (ECPCI), covering ten items in a Likert type, from 1 (always) to 5 (never). The ECPCI afforded excellent internal consistency $(\alpha=0.91)$, and factorial analysis originated two dimensions: annoyance on a daily basis $(\alpha=0.91)$ and criticism and conflicts $(\alpha=0.83)$. The criticism of the caregivers by the-elderly is the highest frequency item, and the lowest was the annoyance in regard to taking care of the elderly. The elderly who do not have partners have more conflicts with the caregivers than the elderly who live with partners $(t=2.76 ; \mathrm{p}<0.05)$. No correlation was found between the level of the caregivers' schooling and their conflicts with the elderly. Finally, strategies are suggested in order to reduce these conflicts: for instance, encouraging organizations to offer courses to caregivers to improve interpersonal communication skills and general ageing knowledge, as well as encouraging care as an emergent and important career in Brazil.
Key words: Interpersonal relationship. Caregivers. Elderly. Conflict scale.

\section{INTRODUÇÃO}

Entre 1998 e 2008, a expectativa de vida no Brasil deu um salto de quase quatro anos, passando de 69 para 73 anos. Hoje o número de pessoas com mais de 60 anos atinge $12 \%$ do total da população brasileira, mas as projeções para 2050 indicam que esta proporção alcançará 22,7\%. ${ }^{1} \mathrm{Um}$ dos maiores desafios da atualidade é manter o bem-estar para essa população que aumenta vertiginosamente.

Do total de idosos brasileiros, um terço reside com seus cônjuges $(33,2 \%)$, quase a metade mora com seus filhos $(44,6 \%), 21,2 \%$ residem com outro parente e $1 \%$ mora com não-parentes. Embora a família ainda represente um recurso importantíssimo para a manutenção do bem-estar da grande maioria dos idosos brasileiros, muitos vivem em instituições de longa permanência, significando $0,8 \%$ da população idosa. ${ }^{2}$ Entretanto, para mantê-los por maior tempo possível no seio familiar, é necessário priorizar o apoio e a instrumentalização da família cuidadora, sobretudo a realização de pesquisas que possam fundamentar propostas e medidas em prol dos idosos e de seus cuidadores em suas residências.
Manter os idosos menos dependentes e ter familiares que possam cuidar dos seus idosos parece ser uma boa estratégia para lidar com o custo do envelhecimento. Segundo Caldas, ${ }^{3}$ a dependência de um indivíduo é caracterizada pela incapacidade de realizar as atividades básicas da vida. A dependência é constituída por um somatório de incapacidades e necessidades dos indivíduos, sendo que estes podem ser modificados se houver condições e assistência adequadas.

As atividades básicas de vida diária são representadas por alimentar-se, vestir-se, controlar os esfíncteres, banhar-se e locomoverse por conta própria. As atividades instrumentais de vida diária são realizar compras, tarefas domésticas, administrar medicações ou manusear dinheiro. As atividades avançadas de vida diária referem-se à automotivação para o trabalho, atividades de lazer, contatos sociais e exercícios físicos. A não-realização dessas atividades pelo idoso pode ser representada por diversos graus de dependência, como independente, leve, parcial ou total. 
Duca, Silva \& $\mathrm{Hallal}^{4}$ constataram que $26,8 \%$ dos idosos de uma pesquisa realizada no município de Pelotas necessitavam de cuidados para atividades básicas de vida diária, ou seja, tinham um grau de dependência parcial e, portanto, os cuidadores os ajudavam a realizar tais atividades. $\mathrm{O}$ cuidador de idoso é alguém, familiar ou não, que oferece assistência e tem a responsabilidade pelo cuidado regular ao idoso. Ainda segundo Leme,${ }^{5}$ o cuidador pode ser formal (profissional) ou informal (familiares e amigos).

A saúde do idoso despertou interesse mundial nas Assembleias Mundiais de Envelhecimento em Viena (1992) e em Madri. Nesta última, a Organização Mundial de Saúde ${ }^{6}$ propôs a Politica de Envelhecimento Ativo, que preconizou, entre outras medidas, a oferta de treinamento e educação para acompanhantes de idosos. Seguindo a proposta de Envelhecimento Ativo da OMS, o Ministério da Saúde lançou, em julho de 2009, o Programa Nacional de Formação de Cuidadores, no qual até 2011 serão treinados 65 mil cuidadores por 36 escolas técnicas do SUS em todo o país. ${ }^{7,8}$

O reconhecimento do cuidador-familiar é uma forma de gratidão ao idoso pela dedicação prévia deste à família. Montezuma, Freitas \& Monteiro 9 apontam que o crescimento pessoal, o senso de autorrealização e o sentimento de gratificação são benefícios decorrentes do ato de cuidar. Contudo, os cuidadores podem apresentar sintomas de estresse e menor nível de satisfação em viver, comparados à população em geral. ${ }^{10}$

Os sintomas de estresse podem ser representados pela sobrecarga de trabalho do cuidador em relação ao idoso. Essa sobrecarga é considerada por Pearlin, Mullan, Semple \& Skaff ${ }^{11}$ como um tipo de estressor primário, assim como os conflitos familiares e os conflitos entre o cuidado e o trabalho do cuidador são considerados estressores secundários. Apesar de os sentimentos negativos existirem na relação cuidador-idoso, estes podem gerar um ônus ou não para o cuidador. Este ônus pode acontecer caso o cuidador apresente baixa resiliência, conflitos e desfuncionalidade em relação ao idoso $^{12}$. Além disso, fatores como gênero, idade, saúde, ocupação, personalidade do cuidador e a história do relacionamento cuidador-idoso podem ser considerados como estressores e interferir também no relacionamento. ${ }^{10}$

$\mathrm{Na}$ tentativa de solucionar a sobrecarga de cuidado, alguns cuidadores familiares estabelecem uma escala de plantão, na qual aqueles que moram próximos quanto os que moram longe assumem tarefas para com os idosos dependentes. Nessa redefinição de papéis e estabelecimento de tempo de dedicação entre os cuidadores familiares, podem ocorrer conflitos intensos de relacionamento. ${ }^{8}$

De acordo com Bengtson, Giarusso, Mabry $\&$ Silverstein, ${ }^{13}$ o conflito faz parte da relação de solidariedade entre pais e filhos, como se fosse sua extensão. Para esses autores, a solidariedade compreende as dimensões do relacionamento que podem ser organizadas de forma que exista uma dialética entre os opostos, tais como intimidade e distância; dependência ou autonomia; integração e isolamento, havendo a formação de diferentes arranjos de relacionamento derivados dessas dimensões. Esses conflitos também são observáveis não apenas entre pais e filhos, mas entre os idosos e seus parceiros cuidadores, que tenham ou não algum grau de parentesco. Nota-se que, na presente pesquisa, muitos idosos eram cuidados por seus filhos (35\%). Na literatura, Torres et al. ${ }^{14}$ encontraram apenas $15,4 \%$. No estudo de Neri et al., 21,6\% dos cuidadores (filhos desses idosos) moravam com os mesmos em 1997. ${ }^{15}$

Após o falecimento do cônjuge do idoso, os filhos têm um sentimento de obrigação filial pelo idoso, com base em sentimentos de amor. ${ }^{16}$ Isto é bastante observado nos países de culturas coletivistas, como os asiáticos e os latinos. Além disso, existe uma relação de reciprocidade entre o filho e o idoso, mais do que propriamente solidariedade. ${ }^{15}$ Quando o cuidador filho apresenta dependência econômica e vive na mesma casa que o idoso, pode ficar estressado, ${ }^{17}$ desencadeando um tipo de conflito como o mautrato ao idoso..$^{18}$ As mulheres cuidadoras relatam mais ônus no cuidado ${ }^{19}$ e quando não assumem o cuidado, podem ser alvo de conflitos familiares. ${ }^{15}$ 
Os estudos sobre conflitos interpessoais entre idosos e cuidadores abrem possibilidades para a adoção de posturas a serem introduzidas na educação escolar, no trabalho, na família, de forma a reduzir os conflitos. Sobretudo, podem fornecer dados para estimular uma relação mais harmoniosa entre cuidadores e idosos. Neste sentido, esta pesquisa priorizou o estudo do relacionamento interpessoal entre idosos e seus cuidadores, familiares ou não, especialmente os conflitos percebidos pelos cuidadores e os primeiros.

Dentre esses estudos, destacam-se a pesquisa de Silverstein, Chen \& Heller, ${ }^{20}$ revelando que o estado civil do idoso e o apoio em excesso recebido por ele estavam correlacionados à maior predisposição para conflitos com seus cuidadores. Kalmijn ${ }^{21}$ ressaltou que os idosos que não tinham companheiros recebiam menor apoio dos cuidadores. Ainda segundo o autor, além do estado civil do idoso, o nível educacional dos cuidadores poderia influenciar o conflito entre eles.

O presente estudo teve por objetivo testar uma escala de conflitos cuidadores-idosos e averiguar a influência do estado civil do idoso e do nível de escolaridade do cuidador, em função dos possíveis conflitos entre eles.

Método

A presente pesquisa adotou a linha quantitativa, utilizando-se de um questionário (anexo 1) que foi preenchido por 100 cuidadores dos idosos, com a assistência do pesquisador principal. Conforme será descrito nos subitens subsequentes, foi construída a Escala de Conflitos no Relacionamento Cuidador-Idoso (ECRCI), avaliada pelos cuidadores. Alguns itens do instrumento de solidariedade entre idosos e cuidadores de Lowestein, Katz, MehlhausenHassoen e Prilutzky ${ }^{22}$ foram adaptados e utilizados para construir e validar a escala ECPCI, tipo Likert, variando de 1 (sempre) a 5 (nunca). Além de testar e validar a ECRCI, esta pesquisa teve como objetivo testar a influência de duas variáveis sociodemográficas, como o estado civil do idoso e o nível de escolaridade do cuidador, nos conflitos observados pelos cuidadores em seu relacionamento com os idosos. As duas hipóteses foram influenciadas pelo trabalho de Kalmijn, ${ }^{21}$ como descritas abaixo. 
QUESTIONÁRIO-Anexo 1

ESCALA DE CONFLITOS PELO CUIDADOR NO SEU RELACIONAMENTO COM O IDOSO -ECPCI

Com que frequência você se aborreceu com este (a) idoso (a) nos últimos 6 meses, em relação a:

$1=$ SEMPRE 2 = QUASE SEMPRE $3=$ ALGUMAS VEZES $4=$ RARAMENTE 5 = NUNCA

1. PEQUENAS IRRITAÇÕES DIÁRIAS (Pa 20)

2. QUESTÕES DE DINHEIRO (Pa 20)

3. RELACIONAMENTO PAIS E FILHOS DO IDOSO (Pa 20)

4. DROGAS, BEBIDAS ALCOÓLICAS OU FUMO (Pa 20)

5. CRENÇAS E VALORES (Pa 20)

6. CUIDADO COM AS DOENÇAS DESTE IDOSO (Pa 20)

7. DEVERES E RESPONSABILIDADES (Pa 20)

8. CONFLITOS E TENSÕES ENTRE VOCÊS (Pa 17)

9. CRITICISMO DO IDOSO EM RELAÇÃO A VOCÊ (Pa 18)

10. DISCUSSÕES ENTRE VOCÊ E O IDOSO (Pa 19)

Fonte: Questões adaptadas do questionário utilizado no Projeto OASIS por Lowestein , Katz , MehlhausenHassoen \& Prilutzky (2005).

Obs. 1: O item 3 se refere aos aborrecimentos do cuidador com a família do idoso. No caso do cuidador ser o filho ou a filha deste idoso, o aborrecimento se refere a outro membro da família.

Obs. 2: Pa 17, Pa18 e Pa 20, referem-se às questões que foram selecionadas e adaptadas do questionário OASIS.

Hipóteses do estudo

Kalmijn ${ }^{21}$ ressaltou que os idosos que não tinham companheiros recebiam menor apoio dos cuidadores. Assim, este estudo verificou se há correlação significativa entre a variável de viver ou não com companheiro e a frequência de conflitos no relacionamento cuidador-idoso, conforme disposto abaixo:

Hipótese 1: Os idosos que não possuem companheiros (as) apresentam maiores níveis de conflito com seus cuidadores do que os idosos que vivem com companheiros.

Kalmijn ${ }^{21}$ assinalou, ainda, que a maior predisposição a conflitos no relacionamento pode ocorrer quando os cuidadores têm alto nível de educação. Assim, este estudo testou a existência de correlação significativa entre o nível de escolaridade dos cuidadores e a frequência dos conflitos dos cuidadoresidosos, conforme hipótese formulada a seguir.

Hipótese 2: Os cuidadores com maior nível de escolaridade apresentam maiores índices de conflito com os idosos do que cuidadores com menor nível de escolaridade.

\section{Participantes}

Participaram deste estudo 100 cuidadores de idosos de 18 anos ou mais, dentre os quais pessoas de três grupos residentes de locais distintos: Paquetá (31 cuidadores), Maricá-Itaipuaçu (47 cuidadores), e outro formado por cuidadores residentes de locais diversos do Estado do Rio de Janeiro (22 cuidadores). A opção pelos dois primeiros bairros se justifica pela conveniência de a pesquisadora trabalhar nos dois respectivos 
postos de saúde, o que de certa forma facilitou o acesso aos cuidadores desses idosos. Entretanto, para completar o número dos participantes necessários para a testagem da escala de dez itens, solicitou-se aos profissionais de saúde de Paquetá e de Maricá que indicassem cuidadores de outros locais para a aplicação dos questionários restantes. Por este motivo, a amostra foi caracterizada como de conveniência.

Os critérios de inclusão dos participantes na pesquisa foram: ser maior de 18 anos e estar cuidando de pelo menos um idoso, familiar ou não deste cuidador. Foram convidados 133 cuidadores que atendiam ao critério de inclusão, mas só aceitaram participar desta pesquisa 100 cuidadores. A média de idade dos cuidadores era de 52 anos, variando entre 18 e 88 anos, e a maioria era do sexo feminino (77\%). Em torno de $65 \%$ eram casados ou viviam juntos. O nível de escolaridade desse grupo foi bem distribuído, com a metade tendo no máximo instrução de nível médio, $23 \%$ possuíam nível técnico, pouco mais de um quarto possuía nível superior, mas $5 \%$ eram analfabetos.

Quanto à situação financeira desses cuidadores, quase a metade declarou estar em situação financeira razoável, apenas um quarto admitiu enfrentar dificuldades financeiras e um quinto estava em situação confortável. A grande maioria (96\%) desses cuidadores não recebia gratificação financeira por ser o cuidador do idoso. Metade dos participantes se declarou como o único cuidador, e pouco mais de um terço dos cuidadores dividiam as tarefas de "cuidar" com outras pessoas. Pouco mais que um décimo (13\%) não se definia como cuidador, mas como acompanhante, embora na interpretação dos autores deste estudo todos desempenhavam a função de cuidar de seus idosos. Aproximadamente metade era casada ou vivia junto com parceiro. Um terço era viúva, e outros $5 \%$, divorciados e solteiros. Estes dados sociodemográficos dos cuidadores estão dispostos na tabela 1. 
Tabela 1 - Variáveis sociodemográficas dos cuidadores. Niterói, RJ, 2010.

\begin{tabular}{|c|c|c|c|c|}
\hline Variáveis & & $\mathrm{F}$ & $\mathrm{F} \%$ & F\% acumulada \\
\hline \multirow[t]{2}{*}{ Sexo } & Feminino & 77 & 77 & 77 \\
\hline & Masculino & 23 & 23 & 100 \\
\hline \multirow[t]{2}{*}{ Idade } & Até 51 anos & 51 & 51 & 50 \\
\hline & 52 ou mais & 48 & 49 & 100 \\
\hline \multirow[t]{5}{*}{ Estado civil } & Casado & 47 & 47 & 47 \\
\hline & vivendo junto & 18 & 18 & 65 \\
\hline & Viúvo & 4 & 4 & 69 \\
\hline & Divorciado & 13 & 13 & 82 \\
\hline & Nunca foi casado & 18 & 18 & 100 \\
\hline \multirow[t]{5}{*}{ Escolaridade } & Analfabeto & 5 & 5 & 5 \\
\hline & Fundamental & 27 & 27 & 32 \\
\hline & Médio & 19 & 19 & 52 \\
\hline & Nível técnico & 22 & 22 & 74 \\
\hline & Nivel superior & 26 & 26 & 100 \\
\hline Situação & Muito difícil & 6 & 6 & 6 \\
\hline \multirow[t]{4}{*}{ Financeira } & Dificuldades & 18 & 18 & 24 \\
\hline & Razoável & 52 & 52 & 76 \\
\hline & Confortável & 23 & 23 & 99 \\
\hline & Muito confortável & 1 & 1 & 100 \\
\hline Estado & Empregado & 33 & 34 & 34 \\
\hline \multirow[t]{5}{*}{ Ocupacional } & Aposentado & 32 & 33 & 67 \\
\hline & Dono(a)-de-casa & 11 & 11 & 78 \\
\hline & Estudante & 3 & 3 & 81 \\
\hline & Conta própria & 15 & 16 & 97 \\
\hline & Outros & 3 & 3 & 100 \\
\hline Carga & Até $20 \mathrm{~h}$ & 58 & 58 & 58 \\
\hline \multirow[t]{2}{*}{ Horária } & $20-39 \mathrm{~h}$ & 10 & 10 & 68 \\
\hline & $40 \mathrm{~h}$ ou mais & 32 & 32 & 100 \\
\hline Número de & Um cuidador & 50 & 50 & 50 \\
\hline \multirow[t]{2}{*}{ Cuidadores } & Mais de um cuidador & 37 & 37 & 87 \\
\hline & Não é cuidador & 13 & 13 & 100 \\
\hline
\end{tabular}

Instrumento

O instrumento de pesquisa foi baseado em um questionário (anexo 1) utilizado pelo projeto OASIS, coordenado pela Dra. Ariella Lowenstein, ${ }^{22}$ que por meio de 54 itens avaliou o relacionamento entre idosos e cuidadores residentes em cinco países da Europa. O questionário utilizado no projeto OASIS foi traduzido para o português pela segunda autora, nativa na língua portuguesa e fluente na língua inglesa. Do instrumento original, foram selecionados e adaptados apenas dez itens que abordavam os conflitos cuidadores-idosos, 
formando, assim, a Escala de Conflitos Percebidos pelo Cuidador no Relacionamento com o Idoso (ECPCI). Logo após a seleção dos itens e tradução para o português, o novo instrumento foi traduzido novamente para a língua inglesa, por um professor de inglês nascido na Inglaterra e residente no Brasil há muitos anos. Para verificar a clareza, o instrumento foi testado com o grupo de pesquisa que a segunda autora coordena na Universidade, e confirmou-se que realmente testava o que se propunha no nível conceitual e semântico. Assim, o instrumento foi aplicado, sob forma de entrevista, pela pesquisadora a 100 cuidadores de idosos, residentes nos bairros Ilha de Paquetá (Rio de Janeiro), Itaipuaçu (Maricá) e, em menor proporção, em outros bairros do município do Rio de Janeiro.

\section{Procedimentos}

O projeto foi submetido ao Comitê de Ética em Pesquisa da Universidade Salgado de Oliveira (UNIVERSO), sendo aprovado sob o número 672008. Além do Comitê de Ética da Universidade, o projeto foi submetido também ao Comitê de Ética em Pesquisa da Secretaria Municipal de Saúde (SMS-RJ), aprovado sob o número 22-09. Os participantes assinaram o termo de consentimento livre e esclarecido, e cada entrevista durou em média 30 minutos.

No bairro Ilha de Paquetá, a pesquisa foi conduzida nos domić́lios dos moradores idosos de três partes do bairro (centro, norte e sul), préselecionados por meio de uma lista de idosos residentes na região. Os participantes foram contatados por telefone pela pesquisadora e convidados para participar, sendo-lhes explicados o objetivo e a relevância da pesquisa. Setenta e três cuidadores foram convidados para participar da pesquisa, mas apenas 38 aceitaram participar. Em função do número reduzido de cuidadores em Paquetá e do tempo exíguo para a realização da coleta de dados, a pesquisa foi também realizada em outro local, e a amostra que pretendia ser representativa dos idosos de Paquetá foi transformada em uma amostra de conveniência. Assim, o segundo local para aplicação de pesquisa foi um Posto de Saúde em Itaipuaçu, onde a pesquisadora também trabalhava.

No bairro de Itaipuaçu, foi obtida a autorização prévia da Superintendência de Saúde Coletiva do município de Maricá e da chefia do posto de saúde local. Os cuidadores dos idosos responderam ao questionário, também sob forma de entrevista, no posto de saúde local, antes ou após terem sido atendidos pelos profissionais do ambulatório e emergência do posto. O tempo de aplicação também foi o mesmo de Paquetá (30 minutos), e igualmente o termo de consentimento livre e esclarecido foi assinado pelos participantes. Dos 60 cuidadores convidados em Itaipuaçú, 47 aceitaram ser entrevistados.

As amostras de Paquetá e a de Itaipuaçu somaram apenas 78 participantes. De modo a testar a escala de frequência de conflitos cuidadoridosos de dez itens (ECPCI), foi preciso obter uma média de dez participantes para cada item. Assim, para completar o mínimo de 100 cuidadores necessários para a análise estatística da pesquisa, foram convidados mais 22 cuidadores, indicados por profissionais da área de saúde que trabalhavam com a pesquisadora. A amostra total foi composta por 100 cuidadores, homens e mulheres, residentes no Rio de Janeiro, com 18 anos ou mais, parentes ou não dos idosos que cuidavam.

\section{Análise estatística}

Concomitantemente à aplicação dos questionários, os dados (escores brutos) foram lançados na base de dados do sistema SPSS, versão 16.0. Para a caracterização da amostra total, foram calculados médias, percentuais e desvios-padrões das variáveis sociodemográficas.

Foram efetuados a análise fatorial e o teste de fidedignidade da escala de conflitos. Para testar as hipóteses, utilizou-se a Correlação de Pearson. Foram então apresentadas as possíveis correlações entre a reciprocidade e o conflito e as variáveis independentes relacionadas aos idosos, como estado civil do idoso e nível de escolaridade do cuidador. O cruzamento das variáveis foi realizado 
com as médias de cada dimensão resultante da análise fatorial das escalas de conflito e de reciprocidade, e suas respectivas dimensões.

Após a inserção dos dados dos questionários no banco de dados, eles foram armazenados em local específico da Universidade e ficarão em poder dos pesquisadores pelos próximos cinco anos, quando serão destruídos.

\section{RESULTADOS}

As características dos participantes foram descritas acima. Abaixo seguem os dados sociodemográficos dos idosos e os demais resultados obtidos nesta pesquisa.

\section{Grupo dos idosos}

A média de idade dos idosos era de 72 anos $(\mathrm{DP}=7,97)$ variando de 59 a 93 anos, e mais da metade era do sexo feminino (55,7\%). A maioria (80\%) dos idosos declarou escolaridade baixa (um décimo de analfabetos e $70 \%$ dos participantes possuía até o ensino fundamental). Da mesma forma que no caso dos cuidadores, aproximadamente metade dos idosos (47\%) apresentou uma situação financeira razoável, cerca de um terço declarou viver confortavelmente, $\mathrm{e}$ apenas um quarto dos idosos (24\%) declarou viver com muita dificuldade financeira.

Pouco mais que a metade (61\%) era casada ou vivia junto com parceiro, um terço era viúvo e apenas $2 \%$ de solteiros. A maioria dos idosos tinha algum grau de parentesco (92\%) com os cuidadores, como parceiros (41\%) e pais dos cuidadores (35\%). Os outros graus de parentesco distribuíam-se entre avós (7\%), irmãs (4\%), outros parentes (3\%), sogros e sogras (2\%). Um percentual reduzido de idosos (8\%) não era parente.
Quanto ao grau de dependência física dos idosos avaliada pelos cuidadores, quase a metade foi avaliada como independente. Entretanto, 29\% destes idosos apresentaram pouco e razoável dependência, e $23 \%$ foram considerados como muito ou extremamente dependentes. Estas categorias de dependência diziam respeito à capacidade do idoso para alimentar-se, vestir- se, controlar os esfíncteres, banhar-se, locomover-se, realizar compras, tarefas domésticas, administrar medicações e manusear dinheiro. Segundo a definição de Caldas, ${ }^{3}$ a dependência de um indivíduo é apontada como a incapacidade de realizar as atividades básicas da vida que são caracterizadas em atividades de vida diária e atividades instrumentais de vida.

As atividades básicas de vida diária são representadas por alimentar-se, vestir-se, controlar os esfíncteres, banhar-se e locomover-se por conta própria. As atividades instrumentais de vida diária são realizar compras, tarefas domésticas, administrar medicações ou manusear o dinheiro. A não realização dessas atividades pelo idoso pode ser representada por diversos graus de dependência como independente, leve, parcial ou total. ${ }^{2}$ Os autores deste artigo decidiram representar os graus de dependência em: independente, de 1 a 5 , sendo $1=$ totalmente dependente (todas as atividades básicas e instrumentais da vida diária eram realizadas com a ajuda do cuidador), 2 = muito dependente (muitas atividades básicas e instrumentais da vida diária eram realizadas com a ajuda do cuidador), 3 $=$ razoavelmente dependente (quando o idoso precisava de ajuda para se vestir ou para tomar um medicamento), 2 = pouco dependente (o idoso precisava apenas de apoio ou companhia para a realização de poucas atividades básicas e instrumentais); e finalmente $5=$ totalmente independente de alguém para realizar as atividades básicas e instrumentais de vida diária. Os dados dos idosos estão dispostos na tabela 2 . 
Tabela 2. Variáveis sociodemográficas e dependência física dos idosos. Niterói, RJ, 2010.

\begin{tabular}{|c|c|c|c|c|}
\hline Variáveis & & $\mathrm{F}$ & $\%$ & \% válidos \\
\hline \multirow[t]{2}{*}{ Idade } & até 71 anos & 48 & 48 & 49 \\
\hline & 72 ou mais & 49 & 52 & 100 \\
\hline \multirow[t]{2}{*}{ Sexo } & masculino & 43 & 46 & 46 \\
\hline & Feminino & 50 & 54 & 100 \\
\hline \multirow[t]{5}{*}{ Estado civil } & Casado & 48 & 48 & 48 \\
\hline & vivendo junto & 13 & 13 & 61 \\
\hline & Viúvo & 34 & 34 & 95 \\
\hline & Divorciado & 3 & 3 & 98 \\
\hline & Solteiro & 2 & 2 & 100 \\
\hline \multirow[t]{6}{*}{ Escolaridade } & Analfabeto & 10 & 10 & 10 \\
\hline & Fundamental & 48 & 49 & 59 \\
\hline & Médio & 21 & 21 & 80 \\
\hline & Nível técnico & 9 & 9 & 89 \\
\hline & Nível superior & 8 & 8 & 97 \\
\hline & Pós-graduação & 3 & 3 & 100 \\
\hline Situação & Muito difícil & 6 & 6 & 6 \\
\hline \multirow[t]{4}{*}{ Financeira } & Dificuldades & 17 & 17 & 23 \\
\hline & Razoável & 47 & 47 & 70 \\
\hline & Confortável & 29 & 29 & 99 \\
\hline & Muito confortável & 1 & 1 & 100 \\
\hline \multirow[t]{10}{*}{ Parentesco } & Esposo & 41 & 41 & 41 \\
\hline & Mãe & 25 & 25 & 66 \\
\hline & Pai & 10 & 10 & 76 \\
\hline & Avô & 1 & 1 & 77 \\
\hline & Avó & 6 & 6 & 83 \\
\hline & Sogro & 1 & 1 & 84 \\
\hline & Sogra & 1 & 1 & 85 \\
\hline & Irmã & 4 & 4 & 89 \\
\hline & Outro parente & 3 & 3 & 92 \\
\hline & Não-parente & 8 & 8 & 100 \\
\hline Dependência & Totalmente dependente & 9 & 9 & 9 \\
\hline \multirow[t]{4}{*}{ Física } & Muito dependente & 14 & 14 & 24 \\
\hline & Razoavelmente & 17 & 17 & 41 \\
\hline & Pouco dependente & 11 & 11 & 52 \\
\hline & Independente & 47 & 49 & 100 \\
\hline
\end{tabular}

Escala de conflitos percebidos pelo cuidador no relacionamento com o idoso (ECPCI)

A ECPCI foi construída para avaliar a frequência dos conflitos percebidos pelo cuidador no seu relacionamento com o idoso. Os itens utilizados e modificados da escala OASIS ${ }^{22}$ relacionados ao conflito foram: $\operatorname{ch} 25$ - conflitos e tensões entre idosos e cuidador; ch26 - quantas vezes este idoso e critico em relação a você; ch27 - 
quantas vezes você discute com seu idoso. E, finalmente, foram selecionados sete dos oito itens da questão ch28 - que avaliava a frequência dos aborrecimentos do cuidador com o idoso. Os sete itens selecionados e adaptados foram pequenas irritações diárias, questões de dinheiro, questões de relacionamento pais e filhos, drogas, bebidas alcoólicas ou fumo, crenças e valores, cuidado com as doenças do idoso(a) e os aborrecimentos relativos aos deveres e responsabilidades deste idoso. Interessante assinalar que o questionário OASIS ${ }^{22}$ utilizou uma escala de $1 \mathrm{a} 6$, que avaliava o conflito em termos de quantidade que se misturava com frequência, ou seja, (1) nenhuma das vezes, (2) um pouco, (3) algumas vezes, (4) muito, (5) bastante, e (6) extremamente. Por outro lado, o instrumento OASIS $^{22}$ avalia a percepção dos idosos e dos cuidadores, e a intenção desta pesquisa foi avaliar apenas a visão do cuidador. Portanto, apenas dez itens foram selecionados e adaptados, e avaliaram a frequência dos conflitos entre cuidador e idoso, em termos de periodicidade, dispostos em tipo Likert, com graduações em ordem decrescente de frequência, variando de 1 - sempre a 5 - nunca (tabela 3). Quanto maior o grau atribuído, menos frequente era o conflito percebido pelo cuidador no seu relacionamento com o idoso.

A média geral da ECPCI apresentada pelos participantes desta pesquisa foi de 3,27 (DP = 1,14). Ou seja, os conflitos observados pelos cuidadores com seus idosos foram considerados razoavelmente frequentes. $\mathrm{O}$ item criticismo do idoso com o cuidador obteve a menor média, representando o maior conflito entre cuidadores e idosos. O item aborrecimentos relativo aos deveres e responsabilidades obteve a maior média, ou seja, representou o menor conflito entre eles.

Tabela 3 - Escala de conflitos percebidos pelo cuidador (ECPCI). Niterói, RJ, 2010.

\begin{tabular}{|c|c|c|c|c|c|c|}
\hline \multirow[t]{2}{*}{ Ítens da escala } & \multirow[t]{2}{*}{$\mathrm{N}$} & \multirow[t]{2}{*}{$\mathrm{M}$} & \multirow[t]{2}{*}{ DP } & \multicolumn{2}{|c|}{$\begin{array}{c}\text { Cargas } \\
\text { Fatoriais }\end{array}$} & \multirow[t]{2}{*}{$\mathrm{h}^{2}$} \\
\hline & & & & D1 & D2 & \\
\hline Aborrecimento por drogas & 93 & 3,62 & 1,82 & 0,86 & 0,19 & 0,78 \\
\hline $\begin{array}{l}\text { Aborrecimento pelas } \\
\text { responsabilidades }\end{array}$ & 93 & 3,67 & 1,67 & 0,85 & 0,24 & 0,78 \\
\hline Aborrecimento por dinheiro & 93 & 3,44 & 1,69 & 0,77 & 0,36 & 0,71 \\
\hline Aborrecimento por crenças/valores & 93 & 3,37 & 1,62 & 0,75 & 0,36 & 0,69 \\
\hline Aborrecimento pelas doenças & 93 & 3,01 & 1,61 & 0,73 & 0,14 & 0,56 \\
\hline Relacionamento pais e filhos & 93 & 3,43 & 1,61 & 0,69 & 0,48 & 0,71 \\
\hline Irritações diárias com o idoso & 93 & 3,10 & 1,34 & 0,47 & 0,45 & 0,42 \\
\hline Criticismo com o cuidador & 93 & 2,83 & 1,21 & 0,12 & 0,84 & 0,71 \\
\hline Discussão com o cuidador & 93 & 3,13 & 1,31 & 0,41 & 0,80 & 0,81 \\
\hline Conflitos e tensões & 93 & 3,08 & 1,14 & 0,25 & 0,78 & 0,67 \\
\hline \multicolumn{4}{|c|}{ Percentual de variância explicada (total $=68,41)$} & 56,96 & 11,43 & \\
\hline \multicolumn{4}{|l|}{ Eigenvalues } & 5,70 & 1,14 & \\
\hline \multicolumn{4}{|c|}{ Coeficiente Alfa de Cronbach (Escala total $\alpha=0,91$ ) } & 0,92 & 0,83 & \\
\hline \multicolumn{4}{|l|}{ Casos válidos } & 93 & 93 & \\
\hline
\end{tabular}

Nota: Esta medida compreende 10 ítens apresentados numa escala Likert, variando de $1=$ sempre a $5=$ nunca. 
Análise fatorial da escala (ECPCI)

Noventa e três cuidadores preencheram todos os itens desta escala, o que significou uma relação aproximada de dez pessoas por item, considerada aceitável à aplicação da técnica análise fatorial..$^{23}$ A ECPCI apresentou o índice de Kaiser-MeyerOlkin (KMO) de 0,89, o que significa que a amostra estava adequada, de acordo com Tabachnick \& Fidel $;{ }^{24}$ e o teste de esfericidade de Bartlett de 558,67 (df = 45; $\mathrm{p}<0,001$ ) apontou para a fatoriabilidade da matriz. Os dados foram submetidos à análise dos componentes principais, com rotação varimax, e seus resultados também reforçados pelo desempenho do scree plot, utilizando-se o critério de eigenvalue maior do que 1. Assim, como pode ser observado na tabela 3, as duas dimensões que obtiveram eigenvalue acima de 1 emergiram da ECPCI e explicaram um total de $68,41 \%$ da variância total da escala.

A primeira dimensão foi nomeada de aborrecimentos no dia a dia, e compreendeu sete itens, a saber: aborrecimentos com drogas, aborrecimentos por responsabilidades e deveres, aborrecimentos por questões de dinheiro, aborrecimento por crenças/valores, aborrecimento por doenças, aborrecimento em relação ao relacionamento pais e filhos e irritações diárias com o idoso, explicando $56,96 \%$ da variância. As cargas fatoriais variaram de 0,47 a 0,86. A média desta dimensão foi de $3,35(\mathrm{DP}=1,31)$.

A segunda dimensão, denominada criticismo e conflitos, foi formada por três itens: criticismo do idoso com o cuidador, discussão do idoso com o cuidador e conflitos/tensões, explicando $11,43 \%$ da variância. As cargas fatoriais variaram de 0,78 a 0,84. A média desta dimensão foi de $3,06(\mathrm{DP}=1,05)$, menor do que a da primeira dimensão, ou seja, a presença do criticismo e dos conflitos foi mais frequente do que de aborrecimentos do dia a dia.

\section{Fidedignidade da escala (ECPCI)}

A média de correlações inter-item da escala ECPCI foi de 0,51, e as correlações do item-total situavam-se entre 0,42 e 0,81. A escala apresentou excelente nível de consistência interna $(\alpha=0,92$; $\mathrm{n}=93$ ). A primeira dimensão aborrecimentos do dia a dia apresentou excelente consistência interna $(\alpha=0,91 ; \mathrm{n}=93)$; com correlações de item-total variando de 0,42 a 0,78. A média das correlações inter-item desta dimensão foi de 0,58. A segunda dimensão criticismo e conflitos apresentou bom índice de confiabilidade $(\alpha=$ $0,83 ; \mathrm{n}=93)$, com correlações de item-total variando de 0,67 a 0,81. A média das correlações inter-item desta dimensão foi de 0,62. A síntese desta escala com seus resultados pode ser observada na tabela 4.

Para a análise da matriz de correlações entre os itens da escala ECPCI, foi utilizada a orientação de Miles \& Shevlin, ${ }^{25}$ que classifica a magnitude dos coeficientes de correlação de acordo com os seguintes intervalos: 0,10 a 0,29 (baixa); 0,30 a 0,49 (moderada) e > 0,50 (elevada),

Tabela 4 - Síntese da escala de conflitos percebidos pelo cuidador (ECPCI) e suas dimensões. Niterói, RJ, 2010.

\begin{tabular}{lcccccc}
\hline $\begin{array}{l}\text { Escala total e suas } \\
\text { dimensões }\end{array}$ & $\mathrm{N}$ & Média & $\mathrm{DP}$ & $\begin{array}{l}\text { Cronbach } \\
\text { Alfa }\end{array}$ & Cargas fatoriais & $\begin{array}{l}\text { Média } \\
\text { inter-item }\end{array}$ \\
\hline ECPCI (total) & 93 & 3,27 & 1,14 & 0,92 & Entre 0,47-0,86 & 0,51 \\
D1 & 93 & 3,35 & 1,31 & 0,91 & Entre 0,47-0,86 & 0,58 \\
D2 & 93 & 3,06 & 1,05 & 0,83 & Entre 0,78- 0,84 & 0,62 \\
\hline
\end{tabular}

Nota: D1-Aborrecimentos no dia a dia (7 itens); D2-Criticismo e conflitos (3 itens). Variações da escala $1=$ sempre a $5=$ nunca. 
Teste das hipóteses da pesquisa

Hipótese 1 - Os idosos que não possuem companheiros(as) apresentam mais conflito com seus cuidadores do que os idosos que vivem com companheiros. Foi realizado o teste $t$ para grupos independentes $(t=2,76 ; p<0,05)$ e esta hipótese foi confirmada. Assim, os idosos que não possuíam companheiros apresentaram maiores índices de conflito no relacionamento com seus cuidadores $(M$ $=2,88 ; \mathrm{SD}=1,32)$ do que os idosos que possuíam uma relação estável $(M=3,52 ; S D=0,93)$.
Hipótese 2: Os cuidadores com maior nível de escolaridade apresentam maiores índices de conflito com os idosos do que cuidadores de menor nível de escolaridade. Esta hipótese não foi confirmada, pois o nível de escolaridade não se correlacionou com o conflito entre cuidadores e idosos, na escala geral $(r=0,14 ; p>0,05 ; n=$ 93), ou nas dimensões D1 ( $r=0,11, p>0,05)$ e D2 $(r=0,69, p>0,05)$.

As correlações referentes às duas hipóteses podem ser observadas na tabela 5 .

Tabela 5 - Correlações entre os conflitos percebidos pelo cuidador ((ECPCI) e suas dimensões e nível de escolaridade dos cuidadores. Niterói, RJ, 2010.

\begin{tabular}{lcccccc}
\hline Variáveis & $\mathrm{N}$ & $\mathrm{M}$ & $\mathrm{DP}$ & 1 & 2 & 3 \\
\hline 1- Escolaridade & 99 & 1,48 & 0,50 & & & \\
2- D1 & 93 & 3,35 & 1,31 & .11 & & \\
3- D2 & 93 & 3,06 & 1,05 & .69 & $.63 * *$ & \\
4- ECPCI & 93 & 3,27 & 1,14 & .14 & $.96 * *$ & $.80 * *$ \\
\hline
\end{tabular}

Nota: D1: Aborrecimentos no dia a dia; D2: Criticismo e conflitos; Variações da escala: $1=$ sempre a $5=$ nunca.

\section{DISCUSSÃO}

O objetivo deste artigo foi apresentar os conflitos de relacionamento dos idosos e seus cuidadores, na percepção deste último, e verificar se o estado civil do idoso e o nível de escolaridade dos cuidadores influenciavam os conflitos entre eles. Foi construída e validada uma escala de conflitos percebidos pelos cuidadores (ECPCI). Dos itens avaliados na escala de conflitos, o criticismo com o cuidador foi o que representou maior conflito, seguido do item aborrecimento por doenças. Os itens que revelaram menor frequência de conflito entre o cuidador e o idoso foram aborrecimento por responsabilidades e aborrecimento pelo uso de drogas. Da análise fatorial da ECPCI, emergiram duas dimensões: a primeira representou os aborrecimentos do dia a dia e a outra dimensão representou o criticismo e os conflitos.

Os aborrecimentos diários podem ser interpretados como uma insatisfação com os cuidados oferecidos ao idoso no cotidiano. Cattani \& Girardon-Perlini ${ }^{16}$ relataram que o sentimento de insatisfação do cotidiano do cuidado pode ocasionar conflitos entre o cuidador e o idoso. A insatisfação do cuidado no cotidiano pode se dever ao despreparo do cuidador em cuidar do idoso, e isto, vinculado à sobrecarga de trabalho do cuidador, pode desencadear o conflito. ${ }^{17}$ No presente estudo, foi encontrada uma média baixa de aborrecimentos diários do cuidador em relação ao idoso.

Das duas hipóteses testadas pela pesquisa, apenas a primeira foi confirmada. Ou seja, que os idosos que não possuíam companheiros(as) apresentaram maior nível de conflito com seus cuidadores do que idosos que viviam com companheiros. Ou seja, o estado civil do idoso está correlacionado tanto na escala geral, quanto nas duas dimensões de conflitos: de aborrecimentos diários e de criticismo e conflitos. Essa hipótese reforçou o resultado encontrado por 
Kalmijn, ${ }^{21}$ segundo qual idosos que não tinham companheiros recebiam menor apoio dos cuidadores.

Vale destacar que as lembranças do relacionamento passado entre idosos e o cuidador exercem influência no tipo de conflito atual entre eles. No estudo de Neri et al., ${ }^{26}$ quase a metade dos cuidadores estudados relatou lembranças ruins no relacionamento passado com o idoso. Este aspecto é importante porque idosos que tiveram relações conflituosas no passado tendiam a suscitar sentimentos negativos pelos cuidadores. ${ }^{26}$ Neri et al. apontam ainda que os sentimentos negativos referentes aos cuidadores, quando relacionados ao domínio social e psicológico, apresentaram percentuais em torno de 44 a $48 \%$, mais baixos quando comparados aos sentimentos positivos relacionados aos cuidadores. ${ }^{26}$ Estes sentimentos negativos incluíam conflitos familiares, com o trabalho, sobrecarga, rejeição $\mathrm{da}$ incapacidade do idoso pelos outros familiares não-cuidadores, compaixão pelo idoso e irreversibilidade da doença. Na presente pesquisa, o conflito dos idosos com seus companheiros apresentou-se menor do que com os nãocompanheiros, e não identificamos se estes nãocompanheiros tinham ou não lembranças dos idosos na relação passada.

O conflito por aborrecimento por doenças foi considerado como o segundo maior tipo de conflito entre o cuidador e o idoso. Nos resultados observados na pesquisa de Neri et al., ${ }^{26}$ os idosos com alta dependência e doenças altamente incapacitantes apresentavam conflito frequentes com a família, mas não com o cuidador.

A segunda hipótese não foi confirmada, visto que a escolaridade dos cuidadores não influenciou a frequência dos conflitos com os idosos, tanto na escala geral quanto nas suas dimensões. Isto contraria o resultado da pesquisa de Kalmijn ${ }^{21}$ como apontado anteriormente, e o estudo de Szydlik, ${ }^{27}$ que apontou a existência de correlação entre a maior escolaridade dos filhos e o maior grau de conflito destes com os pais idosos. Talvez esses resultados tenham sido diferentes porque a maioria dos participantes da nossa pesquisa não era constituída por filhos (35\%), mas por parceiros (41\%), e no estudo de Szydlik, ${ }^{27}$ o conflito foi proveniente apenas do relacionamento com os filhos cuidadores. Outra possível explicação para esta diferença seria porque os cuidadores da nossa pesquisa viviam com os respectivos idosos, diferentemente dos cuidadores investigados por Szydlik, ${ }^{27}$ dos quais nem todos moravam na mesma casa dos idosos.

\section{CONCLUSÃO}

Os resultados desta pesquisa trazem alguns subsídios que podem ajudar a compreender o relacionamento de cuidadores com idosos residentes no Rio de Janeiro. Os conflitos mais frequentes eram o criticismo ao cuidador, que podem ser interpretados como não aceitação do idoso em ser cuidado por aquela pessoa. É importante ressaltar a necessidade da oferta de grupos de discussão e de apoio para cuidadores, preferencialmente coordenados por psicólogos ou assistentes sociais, para serem analisados os aspectos que influem positiva ou negativamente os conflitos, de forma a fortalecer este relacionamento. Este apoio é muito importante para todos os cuidadores, mas se for preciso aplicar um critério de seleção por vaga em algum grupo de apoio psicológico para os cuidadores, aqueles que cuidam de idosos que não vivem com parceiros talvez sejam os prioritários no atendimento.

A amostra foi composta por três grupos de diferente localização (Paquetá, Itaipuaçu e outros bairros do Rio de Janeiro), mas o número de participantes de cada grupo foi insuficiente para uma comparação entre eles. Apesar disto, a amostra como um todo (100 cuidadores) revelou aceitabilidade no tamanho, principalmente levando-se em conta que a escala de frequência de conflitos cuidador-idoso (ECPCI) possui dez itens, e que a proporção foi de aproximadamente dez participantes por item avaliado. A ECPCI apresentou alto nível de confiabilidade e consistência interna. Futuras pesquisas poderão confirmar a validade do instrumento com uma amostra maior, inclusive comparando-se as diferenças entre a percepção de 
conflitos, na visão dos idosos e na visão dos cuidadores. Recomenda-se ainda a comparação entre as diferenças existentes entre os conflitos que são apresentados pelos cuidadores parceiros (a maioria nesta pesquisa) e pelos cuidadores que são os filhos dos idosos, de acordo com o que foi observado na revisão de literatura.

Recomenda-se ainda que a pesquisa seja replicada em outros postos de saúde do Estado do Rio de Janeiro não somente para fins comparativos, mas para investigar alternativas para a prevenção de conflitos em cada representação de posto de saúde. Uma das alternativas é a promoção de atitudes de resiliência para os cuidadores de idosos, de forma a ajudá-los a reconhecer os problemas do lidar com os idosos, comunicá-los abertamente e ter consciência dos recursos e estratégias para resolvê-los. ${ }^{28}$

\section{REFERÊNCIAS}

1. IBGE. Pesquisa Nacional por Amostra de Domicílios (PNAD). Síntese de Indicadores 2009. Rio de Janeiro: IBGE; 2010.

2. Camarano AA, Kanso S, Pasinato MT, Mello JLE. Idosos brasileiros: indicadores de condições de vida e de acompanhamento de políticas. Brasília: Presidência da República; 2009.

3. Caldas CP. Envelhecimento com dependência: responsabilidades e demandas na família. Cad Saud Públic 2005; 19(3): 733-781.

4. Duca GFD, Silva MC, Hallal PC. Incapacidade funcional para atividades básicas e instrumentais da vida diária em idosos. Rev Saúd Pública 2009, 43(5): 796-805.

5. Leme MD. Treinamento de cuidadores de idosos: impacto na sua qualidade de vida e saúde. São Paulo. Tese. [Doutorado em Medicina]-Faculdade de Medicina da Universidade de São Paulo; 2006.

6. Organização Mundial da saúde(OMS). Envejecimiento Activo: um marco político. Rev Esp Geriatr Gerontol 2002; 37: 74-105.

7. Motta LB . Treinamento Interdisciplinar em Saúde do Idoso: um modelo de programa adaptado às especificidades do envelhecimento. Rio de Janeiro: UnATI; 2003

8. Ministério da Saúde (Brasil). Regulamento do Sistema Único de Saúde. Portaria $n^{\circ} 2.048$ abr
É preciso assinalar a importância das pessoas do sexo masculino compartilharem com as mulheres o cuidado com os idosos, já que a maioria dos cuidadores são mulheres. Esta divisão igualitária de responsabilidades deveria ser reforçada pela mídia, diante da manutenção do idoso na família.

Finalmente, deve-se ressaltar incentivo necessário, por parte do governo, para a criação da profissão de cuidador remunerado, profissional importantíssimo frente ao rápido processo de envelhecimento da sociedade, e ainda raro no Brasil, de forma a se estender o atendimento domiciliar a um maior contingente de idosos. Tal medida poderá afastar a necessidade de internação desses idosos, que é extremamente custosa tanto para o contribuinte quanto para o governo.

2009. [Acesso em 30 out 2010]. Disponível em: http://www.revistajuridica.com.br/content/ legislacao.asp?id $=94151$.

9. Montezuma CA, Freitas MC , Monteiro ARM. A família e o cuidado ao idoso dependente: estudo de caso. Rev Eletrônica de Enfermagem 2008; 10 (2):395-404.

10. Karsch U. Idosos dependentes: famílias e cuidadores. Cad Saúde Pública 2003; 19(3): 861866. [Acesso em 30 de out 2010] Disponível em: http://www.scielosp.org/scielo.php?pid = S0102311X2003000300019\&script $=$ sci_abstract\&tlng $=\mathrm{pt}$

11. Pearlin LI, Mullan JT, Semple SH , Skaff MM. Caregiving and the stress process: an overview of concepts and their measures. The Gerontologist 1990;30(5): 583-594.

12. Braithwaite V. Contextual or general stress outcomes: making choices through caregiving appraisals. The Gerontologist 2000; 40(6): 706 717.

13. Bengtson VL, Giarusso R, Mabry JB, Silverstein M. Solidarity, Conflict, and Ambivalence: Complementary or Competing Perspectives on Intergenerational relationships? J Marriage and Family 2002; 64 (3): 568-576.

14. Torres GV, et al. Funcionalidade familiar de idosos dependentes residentes em domicílios. Avaliação psicológica 2009; 8(3) : 415-23 
15. Neri AL, Pinto MEB, Sommerhalder C, Perracini MR , Yuaso DR. As várias faces do cuidado e do bem-estar do cuidador. In: AL Neri. Cuidar de idosos no contexto da família: questões psicológicas. Campinas: Alínea; 2006.

16. Cattani RB, Girardon-Perlini NMO. Cuidar do idoso doente no domicílio na voz de cuidadores familiares. Rev Eletrônica de Enfermagem 2004; 6(2). [Acesso em 03 abr 2011].Disponível em: http//www.revistas.ufg.br/index.php/fen

17. Nascimento LC,et al. Cuidador de ancianos: conocimiento disponible em La basis de datos LILACS. Rev Bras Enferm 2008; 61(4).514-17.

18. Matos AR. Compreensão do fenômeno dos maus-tratos econômicos e emocionais a idosos e a decisão no processo de institucionalização : uma realidade portuguesa. Rev Kairós Gerontologia 2010; 13(2): 57-73.

19. Peter-Davis ND, Moss MS, Pruchno RA. Children-in-law in caregiving families. The Gerontologist 1999; 39(1): 66-75.

20. Silverstein M, Chen X, Heller K. Too much of a good thing? Intergenerational social support and the psychological well-being of older parents. J Marriage and the Family 1996; 58(4): 970-983.

21. Kalmijn M. Gender differences in the effects of divorce: widowhood and remarriage on intergenerational support: does marriage protect fathers? Social Work Research 2007; 85 (3): 1079-1104.
22. Lowestein A, Katz R, Mehlhausen-Hassoen D, Prilutzky D. The Research Instruments in the OASIS Project Old Age and Autonomy: The Role of Service Systems and Intergenerational Family Solidarity . Israel: University of Haifa;2005.

23. Pasquali L. Testes referentes a construto: teoria e modelo de construção. In: L. Pasquali. Instrumentos psicológicos: manual prático de elaboração. Brasília: Lab PAM-IBAAP;1999.

24. Tabachnick BG, Fidel LS. Using multivariate statistics. 4.ed. San Francisco: Allyn \& Bacon; 2001.

25. Miles J , Shevlin M. Applying Regression e Correlation : a guide for students and researchers. London: SAGE Publications; 2001.

26. Neri AL, et al. Avaliação subjetiva da tarefa de cuidar: ônus e benefícios percebidos por cuidadoras familiares de idosos de alta dependência. In: AL Neri. Cuidar de idosos no contexto da família: questões psicológicas. Campinas: Ed Alínea; 2006.

27. Szydlik M. Intergenerational Solidarity and Conflict. J Comparative Family Studies 2008; 39 (1): 97-114.

28. Ravazolla MC. Resiliências Familiares. In: Melillo ENS. Resiliência: descobrindo as próprias fortalezas. Porto Alegre: Artmed; 2005; p 73-85 